\title{
Per-Antenna Rate and Power Control for MIMO Layered Architectures in the Low- and High-Power Regimes
}

\author{
Angel Lozano*
}

September 30, 2009

\begin{abstract}
In a MIMO layered architecture, several codewords are transmitted from a multiplicity of antennas. Although the spectral efficiency is maximized if the rates of these codewords are separately controlled, the feedback rate within the link adaptation loop is reduced if they are constrained to be identical. This poses a direct tradeoff between performance and feedback overhead. This paper provides analytical expressions that quantify the difference in spectral efficiency between both approaches for arbitrary numbers of antennas. Specifically, the characterization takes place in the realm of the low- and high-power regimes via expansions that are shown to have a wide range of validity.

In addition, the possibility of adjusting the transmit power of each codeword individually is considered as an alternative to the separate control of their rates. Power allocation, however, turns out to be inferior to rate control within the context of this problem.
\end{abstract}

Keywords: Spectral Efficiency; Mutual Information; MIMO; Multiantenna; BLAST; PARC; Low-Power, High-Power

${ }^{*}$ Angel Lozano is with Universitat Pompeu Fabra, 08005 Barcelona, Spain. 


\section{Introduction}

The layered architecture is an enticing approach to MIMO (multiple-input multiple-output) communication [1]. At the transmitter, it elicits a very modular structure that accommodates a standard scalar code behind each of the antennas (cf. Fig. 1) while, at the receiver, it enables successive decoding strategies. These features, however, come at the expense of an added burden on the link adaptation loop if the rates of the codewords transmitted from each antenna are to be separately controlled. This added burden can be sidestepped by equating the rates of all the codewords, which lowers the spectral efficiency but renders a link adaptation loop comparable to that of a single-codeword architecture. There is thus a direct tradeoff between spectral efficiency and feedback overhead.

In fact, the layered architecture was initially popularized (under the name Vertical BLAST in its uncoded form [2], and Horizontal BLAST in its coded incarnation [3]) with equalrate codewords. Drawing parallels with the multiple-access channel [4,5], which is an isomorphic problem, it was subsequently recognized that the layered architecture becomes capacity-achieving when the rate of each codeword is separately controlled [6]-[13]. Under the term PARC (per-antenna rate control), this architecture and precoded variants thereof have been discussed in various standardization fora and adopted for single-user MIMO transmission in systems such as 3GPP UMTS [14] and LTE (long-term evolution) [15]. The layered architecture is also one of the MIMO options in IEEE 802.16 WiMAX [16]. The tradeoff between spectral efficiency and feedback overhead is a central issue in these designs, and various compromises have been formulated [17, 18]. (In LTE, for example, only 2 rate-controlled codewords are transmitted when 4 transmit antennas are available [15].)

This paper presents compact analytical expressions that quantify the loss in spectral efficiency, relative to PARC, when the rates of all the codewords are constrained to being equal. The analysis is conducted in the low- and high-power regimes and the resulting expressions characterize the key performance measures therein. (Although, for intermediate power levels, it appears inevitable to have to resort to numerical computations, the transition between the low- and high-power regimes is fairly swift.) These expressions are meant to facilitate the evaluation of the tradeoff between spectral efficiency and feedback overhead, for arbitrary numbers of antennas, in a setting representative of many contemporary 
wireless systems.

\section{Channel Model}

Considering-for the sake of exposition-an OFDM system, the basic baseband MIMO model on a given tone is

$$
\mathbf{y}=\sqrt{g} \mathbf{H} \mathbf{x}+\mathbf{n}
$$

where $\mathbf{x}$ and $\mathbf{y}$ are the transmit and receive vectors with dimensions $n_{\mathrm{T}}$ and $n_{\mathrm{R}}$, respectively, which are the number of transmit and receive antennas. In turn, $\mathbf{n}$ is white Gaussian noise with spectral density $N_{0}=E\left[\|\mathbf{n}\|^{2}\right] / n_{\mathrm{R}}$. The transmit power, $P$, and the bandwidth, $B$, satisfy $P / B=E\left[\|\mathbf{x}\|^{2}\right]$ and we can define a normalized energy per symbol at the receiver

$$
\mathrm{SNR}=g \frac{P / B}{N_{0}} .
$$

The channel is represented by the $n_{\mathrm{R}} \times n_{\mathrm{T}}$ random matrix $\sqrt{g} \mathbf{H}$, where the constant $g$ represents the average channel gain while the entries of $\mathbf{H}$ are unit-variance complex Gaussian scalars. The receiver tracks $\mathbf{H}$ instantaneously by means of reference symbols inserted periodically in time and frequency and, through the link adaptation loop, controls the rate of one or all the codewords. ${ }^{1}$

We further assume the entries of $\mathbf{H}$ to be IID (independent and identically distributed), in accordance with a desirable scenario for single-user MIMO transmission. (Some comments on antenna correlation are put forth in Section VI.) It follows that no precoding is necessary, i.e., each codeword can be mapped directly to a transmit antenna [19].

\section{Performance Measures}

The achievable rate $R$ (bits/s) and the bandwidth $B$ satisfy

$$
R / B=\overline{\mathcal{I}}(\mathrm{SNR})
$$

\footnotetext{
${ }^{1}$ The overhead associated with such reference symbols is not explicitly accounted for.
} 
where $\overline{\mathcal{I}}$ (SNR) denotes the ergodic spectral efficiency (bits/s/Hz). ${ }^{2}$ To evaluate the tradeoffs between $P, R$ and $B$, it is sometimes convenient to express the spectral efficiency, not as function of SNR but rather as function of the normalized energy per bit [21]. At the receiver, specifically,

$$
\frac{E_{b}^{r}}{N_{0}}=\frac{\mathrm{SNR}}{\overline{\overline{\mathcal{I}}(\mathrm{SNR})}}
$$

from which we can express $\overline{\mathcal{I}}\left(\frac{E_{b}^{r}}{N_{0}}\right) \cdot 3$

\section{A Low-Power Regime}

Denoting by $\frac{E_{b}^{r}}{N_{0} \text { min }}$ the minimum energy per bit required for error-free communication and by $S_{0}$ the slope therein in bits $/ \mathrm{s} / \mathrm{Hz} /(3 \mathrm{~dB})$, the low-power spectral efficiency expands as [21]

$$
\overline{\mathcal{I}}\left(\frac{E_{b}^{r}}{N_{0}}\right)=S_{0} \frac{\left.\frac{E_{b}^{r}}{N_{0}}\right|_{\mathrm{dB}}-\frac{E_{b}^{r}}{N_{0}}{ }_{\text {min }} \mid \mathrm{dB}}{3 \mathrm{~dB}}+\epsilon
$$

where $\left.z\right|_{\mathrm{dB}}=10 \log _{10} z$ while $\epsilon$ is a lower-order term. Using (4), we can also relate the spectral efficiency with SNR via

$$
\left.\mathrm{SNR}\right|_{\mathrm{dB}}=\frac{\overline{\mathcal{I}}}{S_{0}} 3 \mathrm{~dB}+10 \log _{10} \overline{\mathcal{I}}+\left.\left({\frac{E_{b}^{r}}{N_{0} \text { min }}}\right)\right|_{\mathrm{dB}}+o(\overline{\mathcal{I}}) .
$$

The two key performance measures at low SNR are thus $\frac{E_{b}^{r}}{N_{0} \min }$ and $S_{0}$.

\section{B High-Power Regime}

At high SNR, the spectral efficiency usually behaves as [22]

$$
\overline{\mathcal{I}}(\mathrm{SNR})=S_{\infty}\left(\frac{\left.\mathrm{SNR}\right|_{\mathrm{dB}}}{3 \mathrm{~dB}}-\mathcal{L}_{\infty}\right)+o(1)
$$

\footnotetext{
${ }^{2}$ In most modern wireless systems, codewords are interleaved over large swaths of bandwidth and over multiple H-ARQ (hybrid automatic repeat request) rounds suitably spaced in time. Altogether, the frequency and time selectivity of the channel that underlies each codeword is large enough to justify the adoption of an ergodic model altogether [20].

${ }^{3}$ Note the slight abuse of notation in indicating by $\overline{\mathcal{I}}(\cdot)$ distinct functions of SNR and of $\frac{E_{b}^{r}}{N_{0}}$.
} 
where $S_{\infty}$ denotes the high-power slope in bits $/ \mathrm{s} / \mathrm{Hz} /(3 \mathrm{~dB})$ while $\mathcal{L}_{\infty}$ represents the highSNR power offset, in 3-dB units, with respect to an unfaded scalar channel.

To relate the high-power spectral efficiency with $\frac{E_{b}^{r}}{N_{0}}$, we can again use (4) to obtain [22]

$$
\left.\frac{E_{b}^{r}}{N_{0}}\right|_{\mathrm{dB}}=\left(\frac{\overline{\mathcal{I}}}{S_{\infty}}+\mathcal{L}_{\infty}\right) 3 \mathrm{~dB}-10 \log _{10} \overline{\mathcal{I}}+o(1) .
$$

The two key performance measures at high SNR are thus $S_{\infty}$, which represents the slope in terms of both SNR and $\frac{E_{b}^{r}}{N_{0}}$ in $3-\mathrm{dB}$ units, and $\mathcal{L}_{\infty}$.

\section{PARC v. H-BLAST}

Let the receiver decode the codewords sequentially in an arbitrary order given by the index $j .{ }^{4}$ With an MMSE (minimum mean-square error) filter applied to whiten the interference from the other yet-to-be-decoded codewords, the spectral efficiency supported by codeword $j$ is then $[4,6]$

$$
\overline{\mathcal{I}}_{j}=E\left[\log _{2}\left(1+\mathbf{h}_{j}^{\dagger}\left(\mathbf{H}_{j} \mathbf{H}_{j}^{\dagger}+\frac{n_{\mathrm{T}}}{\mathrm{SNR}} \mathbf{I}\right)^{-1} \mathbf{h}_{j}\right)\right]
$$

where $\mathbf{h}_{j}$ is the $j$ th column of $\mathbf{H}$ while $\mathbf{H}_{j}=\left[\begin{array}{l}\mathbf{h}_{j+1} \\ \mathbf{h}_{j+2} \ldots \mathbf{h}_{n_{\mathrm{T}}}\end{array}\right]$ has dimensions $n_{\mathrm{R}} \times\left(n_{\mathrm{T}}-j\right)$. Rather than using (9), however, it is often more convenient to express $\overline{\mathcal{I}}_{j}$ as [7]

$$
\overline{\mathcal{I}}_{j}=E\left[\log _{2} \operatorname{det}\left(\mathbf{I}+\frac{\mathrm{SNR}}{n_{\mathrm{T}}} \mathbf{H}_{j-1} \mathbf{H}_{j-1}^{\dagger}\right)\right]-E\left[\log _{2} \operatorname{det}\left(\mathbf{I}+\frac{\mathrm{SNR}_{n_{\mathrm{T}}}}{\mathbf{H}_{j}} \mathbf{H}_{j}^{\dagger}\right)\right] .
$$

With PARC, the rate of each codeword is separately controlled and the aggregate spectral efficiency is $[4,6]$

$$
\begin{aligned}
\overline{\mathcal{I}}_{\mathrm{PARC}}(\mathrm{SNR}) & =\sum_{j=1}^{n_{\mathrm{T}}} \overline{\mathcal{I}}_{j} \\
& =E\left[\log _{2} \operatorname{det}\left(\mathbf{I}+\frac{\mathrm{SNR}}{n_{\mathrm{T}}} \mathbf{H} \mathbf{H}^{\dagger}\right)\right]
\end{aligned}
$$

${ }^{4}$ In contrast with non-ergodic settings, where the decoding order may have an impact even in IID channels [23], in our setting it is immaterial to PARC. 
which equals the capacity of the channel. Invoking the closed-form expression for (12) given in [24],

$$
\begin{aligned}
\overline{\mathcal{I}}_{\mathrm{PARC}}(\mathrm{SNR})= & \log _{2}(e) e^{n_{\mathrm{T}} / \mathrm{SNR}} \sum_{i=0}^{m-1} \sum_{j=0}^{i} \sum_{\ell=0}^{2 j}\left[\left(\begin{array}{c}
2 i-2 j \\
i-j
\end{array}\right)\left(\begin{array}{c}
2 j+2 n-2 m \\
2 j-\ell
\end{array}\right)\right. \\
& \left.\cdot \frac{(-1)^{\ell}(2 j) !(n-m+\ell) !}{2^{2 i-\ell} j ! \ell !(n-m+j) !} \sum_{q=0}^{n-m+\ell} E_{q+1}\left(\frac{n_{\mathrm{T}}}{\mathrm{SNR}}\right)\right]
\end{aligned}
$$

where

$$
\begin{aligned}
m & =\min \left(n_{\mathrm{T}}, n_{\mathrm{R}}\right) \\
n & =\max \left(n_{\mathrm{T}}, n_{\mathrm{R}}\right) .
\end{aligned}
$$

and $E_{q}(\cdot)$ is the exponential integral of order $q$,

$$
E_{q}(\zeta)=\int_{1}^{\infty} t^{-q} e^{-\zeta t} d t
$$

With the rate of all codewords constrained to being identical, on the other hand, the aggregate H-BLAST spectral efficiency is

$$
\overline{\mathcal{I}}_{\mathrm{H}-\mathrm{BL}}(\mathrm{SNR})=n_{\mathrm{T}} \min _{j}\left\{\overline{\mathcal{I}}_{j}\right\}
$$

which, using (10) and the right-hand side of (13) with a proper choice of $n_{\mathrm{T}}$ and $n_{\mathrm{R}}$, can also be computed in closed form.

Although $\overline{\mathcal{I}}_{\text {PARC }}(\mathrm{SNR}) \geq \overline{\mathcal{I}}_{\mathrm{H}-\mathrm{BL}}(\mathrm{SNR})$, achieving the former requires feeding back $n_{\mathrm{T}}$ times as many coefficients. The expressions we will furnish next provide insights into the difference between these spectral efficiencies.

\section{A Low-Power Regime}

PARC is capacity-achieving and its performance measures are well established. The lowpower performance measures are [21]

$$
\begin{aligned}
\frac{E_{b}^{r}}{N_{0 \text { min }}} & =\frac{1}{\log _{2} e} \\
S_{0} & =\frac{2 n_{\mathrm{T}} n_{\mathrm{R}}}{n_{\mathrm{T}}+n_{\mathrm{R}}}
\end{aligned}
$$


where $\left.\left(1 / \log _{2} e\right)\right|_{\mathrm{dB}}=-1.59$.

Proposition 1 For H-BLAST in a Rayleigh-faded spatially IID channel,

$$
\begin{aligned}
\frac{E_{b}^{\mathrm{r}}}{N_{0 \text { min }}} & =\frac{1}{\log _{2} e} \\
S_{0} & =\frac{2 n_{\mathrm{T}} n_{\mathrm{R}}}{2 n_{\mathrm{T}}+n_{\mathrm{R}}-1}
\end{aligned}
$$

Proof: See Appendix A.

Shown in Figs. 2 and 3 are the low-power expansions for PARC and H-BLAST with, respectively, $n_{\mathrm{T}}=n_{\mathrm{R}}=4$ and $n_{\mathrm{T}}=4, n_{\mathrm{R}}=6$. Also shown are the corresponding exact values obtained via (13), which are in close agreement with the expansions for a wide range of spectral efficiencies.

Since $\frac{E_{b}^{r}}{N_{0} \min }$ is unchanged, the suboptimality of H-BLAST in this regime arises as a diminished slope. The ratio between the slopes, therefore, directly measures the increase in the bandwidth required to achieve a certain rate with a given power, i.e.,

$$
\frac{B_{\mathrm{H}-\mathrm{BL}}}{B_{\mathrm{PARC}}} \approx \frac{2 n_{\mathrm{T}}+n_{\mathrm{R}}-1}{n_{\mathrm{T}}+n_{\mathrm{R}}} .
$$

Example 1 For $n_{\mathrm{T}}=n_{\mathrm{R}}=4$, as in Fig. 2, H-BLAST requires about $37.5 \%$ more bandwidth than PARC in the low-power regime. For $n_{\mathrm{T}}=4, n_{\mathrm{R}}=6$, as in Fig. 3, the increase is about $30 \%$.

The bandwidth penalty is largest for $n_{\mathrm{T}}=n_{\mathrm{R}}$ and it vanishes as $n_{\mathrm{R}} / n_{\mathrm{T}}$ grows large. For $n_{\mathrm{T}}=n_{\mathrm{R}}=n$, it approaches $50 \%$ with growing $n$.

The increase in power required by H-BLAST to attain a rate $R$ with bandwidth $B$, in turn, can be formulated from (6) and Proposition 1 as

$$
\left.\frac{P_{\mathrm{H}-\mathrm{BL}}}{P_{\mathrm{PARC}}}\right|_{\mathrm{dB}} \approx \frac{n_{\mathrm{T}}-1}{2 n_{\mathrm{T}} n_{\mathrm{R}}} \frac{R}{B} 3 \mathrm{~dB}
$$

Example 2 For $n_{\mathrm{T}}=n_{\mathrm{R}}=4$, achieving $R / B=2$ bits $/ \mathrm{s} / \mathrm{Hz}$ with $\mathrm{H}$-BLAST requires $0.56 \mathrm{~dB}$ more power than with PARC.

The rate loss with given power and bandwidth can also be assessed from (6), (19) and Proposition 1. 


\section{B High-Power Regime}

PARC conforms to the usual high-power behavior in (7) irrespective of the relative values of $n_{\mathrm{T}}$ and $n_{\mathrm{R}}$ and, since $\mathbf{H}$ is full rank with probability 1, the high-power slope is $S_{\infty}=$ $\min \left(n_{\mathrm{T}}, n_{\mathrm{R}}\right)$. The power offset, in turn, is

$$
\mathcal{L}_{\infty}=\log _{2} n_{\mathrm{T}}+\left(\gamma-\sum_{\ell=1}^{n-m} \frac{1}{\ell}-\frac{n}{m} \sum_{\ell=n-m+1}^{n} \frac{1}{\ell}+1\right) \log _{2} e
$$

where $\gamma=0.5772$ is the Euler-Mascheroni constant whereas $m$ and $n$ are as defined in (14).

H-BLAST, on the other hand, exhibits markedly different high-power behaviors depending on the relative values of $n_{\mathrm{T}}$ and $n_{\mathrm{R}}$. For $n_{\mathrm{T}}>n_{\mathrm{R}}$, the spectral efficiency is bounded for SNR $\rightarrow$ $\infty$. In essence, the first codeword to be decoded is overwhelmed by interference that the MMSE filter, short of spatial dimensions, is unable to reject. This limitation, unnoticed when the noise dominates (i.e., at low SNR), becomes acute as the SNR grows and the interference becomes dominant.

Proposition 2 Consider H-BLAST in a Rayleigh-faded spatially IID channel with $n_{\mathrm{T}}>n_{\mathrm{R}}$. For large SNR,

$$
\overline{\mathcal{I}}_{\mathrm{H}-\mathrm{BL}}(\mathrm{SNR})=\sum_{\ell=1}^{n_{\mathrm{R}}} \frac{n_{\mathrm{T}}}{n_{\mathrm{T}}-\ell} \log _{2} e+o(1)
$$

and hence $S_{\infty}=0$.

Proof: See Appendix B.

It is therefore clearly undesirable to operate H-BLAST with $n_{\mathrm{T}}>n_{\mathrm{R}}$ beyond the low-power regime.

The situation is radically different for $n_{\mathrm{T}} \leq n_{\mathrm{R}}$. Once the receiver has sufficient dimensionality, the spectral efficiency grows as in (7) with $S_{\infty}=n_{\mathrm{T}}$. The suboptimality is then mirrored in the power offset.

Proposition 2 is thus seen to quantify a notion that is, qualitatively speaking, broadly understood. 
Proposition 3 For H-BLAST in a Rayleigh-faded spatially IID channel with $n_{\mathrm{T}} \leq n_{\mathrm{R}}$, the spectral efficiency behaves as (7) with $S_{\infty}=n_{\mathrm{T}}$ and with

$$
\mathcal{L}_{\infty}=\log _{2} n_{\mathrm{T}}+\left(\gamma-\sum_{\ell=1}^{n_{\mathrm{R}}-n_{\mathrm{T}}} \frac{1}{\ell}\right) \log _{2} e
$$

Proof: See Appendix C.

Shown in Figs. 4 and 5 are the high-power expansions for PARC and H-BLAST with, respectively, $n_{\mathrm{T}}=n_{\mathrm{R}}=4$ and $n_{\mathrm{T}}=4, n_{\mathrm{R}}=6$. Also shown are the corresponding exact values obtained via (13), which are in close agreement beyond some reasonable SNR levels.

Since $S_{\infty}$ is unchanged, the suboptimality of H-BLAST in this regime adopts the form of an increased offset. Such increase immediately measures the additional power required to support a given rate $R$ with fixed bandwidth $B$, i.e.,

$$
\left.\frac{P_{\mathrm{H}-\mathrm{BL}}}{P_{\mathrm{PARC}}}\right|_{\mathrm{dB}} \approx \log _{2}(e)\left(\frac{n_{\mathrm{R}}}{n_{\mathrm{T}}} \sum_{\ell=n_{\mathrm{R}}-n_{\mathrm{T}}+1}^{n_{\mathrm{R}}} \frac{1}{\ell}-1\right) 3 \mathrm{~dB} .
$$

Example 3 For $n_{\mathrm{T}}=n_{\mathrm{R}}=4$, as in Fig. 4, H-BLAST requires $4.7 \mathrm{~dB}$ more power in the high-power regime. For $n_{\mathrm{T}}=4$ and $n_{\mathrm{R}}=6$, as in Fig. 5, the increase is $1.85 \mathrm{~dB}$.

Using the large- $n$ asymptote [25]

$$
\sum_{\ell=1}^{n} \frac{1}{\ell}=\log _{e} n+\gamma+\mathcal{O}(1 / n)
$$

it is found that, for $n_{\mathrm{T}}=n_{\mathrm{R}}=n$ and growing $n$, the power penalty in H-BLAST with respect to PARC approaches

$$
\left.\frac{P_{\mathrm{H}-\mathrm{BL}}}{P_{\mathrm{PARC}}}\right|_{\mathrm{dB}} \approx\left(\log _{2} n-(1-\gamma) \log _{2} e\right) 3 \mathrm{~dB} .
$$

For growing $n_{\mathrm{R}} / n_{\mathrm{T}}$, on the other hand, the power penalty vanishes.

The increase in bandwidth with fixed power and rate, and the rate loss with fixed power and bandwidth, can also be asserted by elaborating on Proposition 3 using (8). 


\section{Optimum Power Allocation for H-BLAST}

In our ergodic setting, the suboptimality of H-BLAST stems from the constraint of operating all the codewords at the same rate regardless of the position of each one in the decoding process, i.e., regardless of the distinct level of interference from other codewords suffered by each one. In contrast, PARC adjusts the codeword rates appropriately. A plausible alternative to the control of the rates is the allocation of unequal transmit powers to the codewords, subject to the applicable power constraint. Intuitively, the performance of H-BLAST is likely to improve if the codewords that are to be decoded early are allocated more power than those that come later in the process. (For PARC, in contrast, the optimum power allocation in a spatially IID channel is uniform [19] and thus there is nothing to be gained.)

Deferring to Section VI the discussion of the feedback and implementation issues, we next concentrate on determining the optimum power allocation for H-BLAST and on evaluating the corresponding spectral efficiency. The analytical tools that we apply are the same utilized thus far.

When the power allocation is not uniform, (9) generalizes to

$$
\overline{\mathcal{I}}_{j}=E\left[\log _{2}\left(1+p_{j} \mathbf{h}_{j}^{\dagger}\left(\mathbf{H}_{j} \mathbf{P}_{j} \mathbf{H}_{j}^{\dagger}+\frac{n_{\mathrm{T}}}{\mathrm{SNR}} \mathbf{I}\right)^{-1} \mathbf{h}_{j}\right)\right]
$$

where $p_{j}$ is the normalized power allocated to codeword $j$ and $\mathbf{P}_{j}=\operatorname{diag}\left\{p_{j+1}, \ldots, p_{n_{\mathrm{T}}}\right\}$. The complete power allocation is described by $\mathbf{P}=\operatorname{diag}\left\{p_{1}, \ldots, p_{n_{\mathrm{T}}}\right\}$, which, under the normalization we have adopted, satisfies $\operatorname{Tr}\{\mathbf{P}\}=n_{\mathrm{T}}$. The spectral efficiency of H-BLAST is then

$$
\overline{\mathcal{I}}_{\mathrm{H}-\mathrm{BL}}(\mathrm{SNR})=n_{\mathrm{T}} \max _{\mathbf{P}: \operatorname{Tr}\{\mathbf{P}\}=n_{\mathrm{T}}} \min _{j}\left\{\overline{\mathcal{I}}_{j}(\mathrm{SNR})\right\}
$$

and the optimum power allocation, $\mathbf{P}^{\star}=\operatorname{diag}\left\{p_{1}^{\star}, \ldots, p_{n_{\mathrm{T}}}^{\star}\right\}$, is the argument of this optimization. As intuition would have it, good power allocation solutions to this max-min problem will seek to equalize the rates supported by the various codewords. 


\section{A Low-Power Regime}

At low SNR, the spectral efficiency is limited by noise rather than by interference between codewords. Since the goal of the power allocation for H-BLAST is precisely to balance such interference, one would expect little if any advantage in departing from a uniform allocation in this regime. Indeed, this is the case.

Proposition 4 For SNR $\rightarrow 0$, the optimum power allocation for $H$-BLAST approaches $\mathbf{P}^{\star}=\mathbf{I}$.

Proof: See Appendix D.

The low-power analysis in Section IV is therefore still applicable.

\section{B High-Power Regime}

At high SNR, in contrast, interference dominates the spectral efficiency and a proper power allocation can lessen the interference that codewords decoded early suffer from those decoded later.

Proposition 5 Let $n_{\mathrm{T}} \leq n_{\mathrm{R}}$. For SNR $\rightarrow \infty$, the optimum power allocation for H-BLAST in a spatially IID channel approaches, in 3-dB units,

$$
\log _{2} p_{j}^{\star}=-\log _{2}\left(\frac{1}{n_{\mathrm{T}}} \sum_{u=1}^{n_{\mathrm{T}}} \exp \left\{-\sum_{\ell=1}^{n_{\mathrm{R}}-n_{\mathrm{T}}+u-1} \frac{1}{\ell}\right\}\right)-\log _{2}(e) \sum_{\ell=1}^{n_{\mathrm{R}}-n_{\mathrm{T}}+j-1} \frac{1}{\ell}
$$

for $j=1, \ldots, n_{\mathrm{T}}$. The corresponding spectral efficiency behaves as (7) with $S_{\infty}=n_{\mathrm{T}}$ and with

$$
\mathcal{L}_{\infty}=\gamma \log _{2} e+\log _{2}\left(\sum_{u=1}^{n_{\mathrm{T}}} \exp \left\{-\sum_{\ell=1}^{n_{\mathrm{R}}-n_{\mathrm{T}}+u-1} \frac{1}{\ell}\right\}\right) .
$$

Proof: See Appendix E.

Presented in Figs. 6 are the exact spectral efficiencies of PARC and H-BLAST with $n_{\mathrm{T}}=n_{\mathrm{R}}=$ 4 and uniform power allocations. In the same plot, we find the exact spectral efficiency of H-BLAST with the optimum power allocation given in Proposition 5. Notice how, as SNR 
grows, such power allocation becomes progressively more effective and the gap with PARC shrinks towards its asymptotic value.

Example 4 Recall that, for $n_{\mathrm{T}}=n_{\mathrm{R}}=4, H$-BLAST with a uniform power allocation requires $4.7 d B$ more power than PARC in the high-power regime (cf. Example 3). From Proposition 5, the optimum transmit powers are

$$
\begin{aligned}
& p_{1}^{\star}=2.28 \\
& p_{2}^{\star}=0.84 \\
& p_{3}^{\star}=0.51 \\
& p_{4}^{\star}=0.37 .
\end{aligned}
$$

and the corresponding deficit dwindles to $1.1 \mathrm{~dB}$ (cf. Fig. 6).

Although the power offset of H-BLAST can be reduced significantly by optimizing the power allocation, as the above example illustrates, it always remains larger than the one for PARC given in (24). (Only for $n_{\mathrm{R}} / n_{\mathrm{T}} \rightarrow \infty$ do they approach the same value.)

\section{Conclusion}

The analysis presented in this paper sets the stage for simple evaluations of the tradeoff between the PARC and H-BLAST layered architectures in an ergodic setting. Although OFDM signaling was invoked for the sake of exposition, the analysis holds for other waveforms as well.

The spectral efficiency shortfall of H-BLAST, which is largest for $n_{\mathrm{T}}=n_{\mathrm{R}}$ and diminishes with growing $n_{\mathrm{R}} / n_{\mathrm{T}}$, comes in exchange for reduced feedback within the link adaptation loop. Take an exemplary configuration with $n_{\mathrm{T}}=n_{\mathrm{R}}=4$. H-BLAST incurs a $37 \%$ increase in bandwidth at low SNR and a 4.7-dB increase in power at high SNR with respect to PARC, but H-BLAST requires feeding back only $25 \%$ of the coefficients needed by PARC. (Depending on the span of the codewords and on the number of bits used to quantize their rates, this converts into a specific difference in the respective feedback bit rates.) For $n_{\mathrm{T}}$ and $n_{\mathrm{R}}$ sufficiently similar, the advantage of PARC appears to warrant this additional feedback. 
We have also seen that optimizing the transmit powers in H-BLAST, while helpful, cannot close the gap with PARC (except for $n_{\mathrm{R}} / n_{\mathrm{T}} \rightarrow \infty$ ). Rate feedback is thus more effective than power feedback in this case.

It is worth noting that, in a true ergodic setting, feedback would theoretically not be necessary in the first place. The codeword rates could be set on the basis of only the channel statistics and the agreed-upon decoding order at the receiver. Actual systems, however, must be robust and designed for general channel conditions. Hence, while the ergodic setting is representative of many scenarios of interest, it cannot be used as an argument to forgo the link adaptation loop.

For channels exhibiting substantial antenna correlation, the analysis is certain to become more involved. Nonetheless, the low- and high-power spectral efficiency of PARC in ergodic channels with antenna correlations is well characterized [26, 27] and there is reasonable hope that the same tools could serve to assert the spectral efficiency of H-BLAST. In fact, if the signaling takes place along the eigenvectors of $E\left[\mathbf{H}^{\dagger} \mathbf{H}\right]$, transmit antenna correlations are isomorphic with non-uniform power allocations [28, 29] and thus the analysis in Section $V$ can be leveraged directly. Also, exact characterizations for various correlation structures of interest have been put forth (e.g., [30]-[33]). Note that, in addition to impacting the spectral efficiency, correlation impacts the feedback load since it opens the door to vector quantization of the channel coefficients.

\section{Acknowledgment}

The author gratefully acknowledges the feedback provided by the reviewers and by the associate editor, which helped improve the paper. 


\section{Appendices}

\section{A Proof of Proposition 1}

In an ergodic setting, the codeword that dominates the spectral efficiency in (17) is the first to be decoded, i.e., $\overline{\mathcal{I}}_{\mathrm{H}-\mathrm{BL}}(\mathrm{SNR})=n_{\mathrm{T}} \overline{\mathcal{I}}_{1}$ (SNR) because codewords $j=2, \ldots, n_{\mathrm{T}}$ suffer from a reduced level of interference. Since all codewords must be encoded at the same rate in H-BLAST, they must all share the rate of that first-decoded one. Thus, using (10) and (17),

$$
\overline{\mathcal{I}}_{\mathrm{H}-\mathrm{BL}}(\mathrm{SNR})=n_{\mathrm{T}} E\left[\log _{2} \operatorname{det}\left(\mathbf{I}+\frac{\mathrm{SNR}}{n_{\mathrm{T}}} \mathbf{H H}^{\dagger}\right)\right]-n_{\mathrm{T}} E\left[\log _{2} \operatorname{det}\left(\mathbf{I}+\frac{\mathrm{SNR}}{n_{\mathrm{T}}} \mathbf{H}_{1} \mathbf{H}_{1}^{\dagger}\right)\right]
$$

where, recall, $\mathbf{H}_{1}=\left[\begin{array}{llll}\mathbf{h}_{2} & \mathbf{h}_{3} & \ldots \mathbf{h}_{n_{\mathrm{T}}}\end{array}\right]$. In conjunction with

$$
\left.\frac{d}{d u} \log _{e} \operatorname{det}(\mathbf{I}+u \mathbf{A})\right|_{u=0}=\operatorname{Tr}\{\mathbf{A}\}
$$

(34) yields

$$
\begin{aligned}
\dot{\overline{\mathcal{I}}}_{\mathrm{H}-\mathrm{BL}}(0) & =\left.\frac{d \overline{\mathcal{I}}_{\mathrm{H}-\mathrm{BL}}(\mathrm{SNR})}{d \mathrm{SNR}}\right|_{\mathrm{SNR}=0} \\
& =n_{\mathrm{R}} \log _{2} e
\end{aligned}
$$

as in PARC. Similarly, using (34) and

$$
\left.\frac{\mathrm{d}^{2}}{\mathrm{~d} u^{2}} \log _{e} \operatorname{det}(\mathbf{I}+u \mathbf{A})\right|_{u=0}=-\operatorname{Tr}\left\{\mathbf{A}^{2}\right\}
$$

we find

$$
\begin{aligned}
\ddot{\mathcal{I}}_{\mathrm{H}-\mathrm{BL}}(0) & =\left.\frac{d^{2} \overline{\mathcal{I}}_{\mathrm{H}-\mathrm{BL}}(\mathrm{SNR})}{d \mathrm{SNR}^{2}}\right|_{\mathrm{SNR}=0} \\
& =-\frac{n_{\mathrm{R}}\left(2 n_{\mathrm{T}}+n_{\mathrm{R}}-1\right)}{n_{\mathrm{T}}} \log _{2} e .
\end{aligned}
$$

Applying [21]

$$
\begin{aligned}
{\frac{E_{b}^{r}}{N_{0 \text { min }}}}=\frac{1 / g}{\dot{\overline{\mathcal{I}}}_{\mathrm{H}-\mathrm{BL}}(0)} \\
S_{0}=-\frac{2}{\log _{2} e} \frac{\left[\dot{\overline{\mathcal{I}}}_{\mathrm{H}-\mathrm{BL}}(0)\right]^{2}}{\ddot{\overline{\mathcal{I}}}_{\mathrm{H}-\mathrm{BL}}(0)},
\end{aligned}
$$

the expressions in the proposition are readily obtained. 


\section{B Proof of Proposition 2}

From (34), canceling a factor $\log _{2} \operatorname{det}\left(\mathrm{SNR} / n_{\mathrm{T}} \cdot \mathbf{I}\right)$ from either expectation,

$$
\begin{aligned}
\overline{\mathcal{I}}_{\mathrm{H}-\mathrm{BL}}(\mathrm{SNR}) & =n_{\mathrm{T}} E\left[\log _{2} \operatorname{det}\left(\mathbf{H H}^{\dagger}+\frac{n_{\mathrm{T}}}{\mathrm{SNR}} \mathbf{I}\right)\right]-n_{\mathrm{T}} E\left[\log _{2} \operatorname{det}\left(\mathbf{H}_{1} \mathbf{H}_{1}^{\dagger}+\frac{n_{\mathrm{T}}}{\mathrm{SNR}} \mathbf{I}\right)\right] \\
& =n_{\mathrm{T}} E\left[\log _{2} \operatorname{det}\left(\mathbf{H H}^{\dagger}\right)\right]-n_{\mathrm{T}} E\left[\log _{2} \operatorname{det}\left(\mathbf{H}_{1} \mathbf{H}_{1}^{\dagger}\right)\right]+o(1) \\
& =n_{\mathrm{T}} \sum_{\ell=0}^{n_{\mathrm{R}}-1} \frac{1}{n_{\mathrm{T}}-\ell-1} \log _{2} e+o(1)
\end{aligned}
$$

where we have used [34]

$$
E\left[\log _{e} \operatorname{det}\left(\mathbf{W} \mathbf{W}^{\dagger}\right)\right]=-n \gamma+\sum_{\ell=0}^{n-1} \sum_{q=1}^{m-\ell-1} \frac{1}{q}
$$

for an $n \times m$ matrix $\mathbf{W}$ with IID zero-mean unit-variance complex Gaussian random entries $(m \geq n)$. Note that $\mathbf{H}$ is $n_{\mathrm{R}} \times n_{\mathrm{T}}$ while $\mathbf{H}_{1}$ is $n_{\mathrm{R}} \times\left(n_{\mathrm{T}}-1\right)$. By shifting the summation index in (45), the claimed expression is finally obtained.

\section{Proof of Proposition 3}

Rearranging (34) in a form that is more convenient for $n_{\mathrm{T}} \leq n_{\mathrm{R}}$

$$
\overline{\mathcal{I}}_{\mathrm{H}-\mathrm{BL}}(\mathrm{SNR})=n_{\mathrm{T}} E\left[\log _{2} \operatorname{det}\left(\mathbf{I}+\frac{\mathrm{SNR}}{n_{\mathrm{T}}} \mathbf{H}^{\dagger} \mathbf{H}\right)\right]-n_{\mathrm{T}} E\left[\log _{2} \operatorname{det}\left(\mathbf{I}+\frac{\mathrm{SNR}}{n_{\mathrm{T}}} \mathbf{H}_{1}^{\dagger} \mathbf{H}_{1}\right)\right] .
$$

The corresponding power offset is then obtained as

$$
\begin{aligned}
\mathcal{L}_{\infty} & =\lim _{\mathrm{SNR} \rightarrow \infty}\left(\log _{2} \mathrm{SNR}-\frac{\overline{\mathcal{I}}_{\mathrm{H}-\mathrm{BL}}(\mathrm{SNR})}{S_{\infty}}\right) \\
& =\log _{2} n_{\mathrm{T}}-E\left[\log _{2} \operatorname{det}\left(\mathbf{H}^{\dagger} \mathbf{H}\right)\right]+E\left[\log _{2} \operatorname{det}\left(\mathbf{H}_{1}^{\dagger} \mathbf{H}_{1}\right)\right]
\end{aligned}
$$

where, in (49), we have used $S_{\infty}=n_{\mathrm{T}}$. Application of (46) to the two expectations leads to the claimed expression. Note, as in Appendix $\mathbf{B}$, that $\mathbf{H}$ is $n_{\mathrm{R}} \times n_{\mathrm{T}}$ while $\mathbf{H}_{1}$ is $n_{\mathrm{R}} \times\left(n_{\mathrm{T}}-1\right)$. 


\section{Proof of Proposition 4}

Rewriting (30) in a more convenient form, as done in (34) for the case of a uniform power allocation,

$$
\begin{aligned}
\overline{\mathcal{I}}_{j}(\mathrm{SNR})= & E\left[\log _{2} \operatorname{det}\left(\mathbf{I}+\frac{\mathrm{SNR}}{n_{\mathrm{T}}} \mathbf{H}_{j-1} \mathbf{P}_{j-1} \mathbf{H}_{j-1}^{\dagger}\right)\right] \\
& -E\left[\log _{2} \operatorname{det}\left(\mathbf{I}+\frac{\mathrm{SNR}}{n_{\mathrm{T}}} \mathbf{H}_{j} \mathbf{P}_{j} \mathbf{H}_{j}^{\dagger}\right)\right]
\end{aligned}
$$

which must be plugged into (31). While, with a uniform power allocation, the limiting codeword is always the first one to be decoded, that need not be the case when the powers are optimized and thus the inner minimization no longer trivially returns $j=1$.

From (41), only the first-order term of $\overline{\mathcal{I}}_{\mathrm{H}-\mathrm{BL}}(\mathrm{SNR})$ is relevant in terms of $\frac{E_{b}^{r}}{N_{0}}$. Applying (35), $\frac{E_{b}^{r}}{N_{0}}$ min is minimized by

$$
\begin{aligned}
\mathbf{P}^{\star} & =\arg \max _{\mathbf{P}: \operatorname{Tr}\{\mathbf{P}\}=n_{\mathrm{T}}} \min _{j}\left\{p_{j} n_{\mathrm{R}} \log _{2} e\right\} \\
& =\mathbf{I} .
\end{aligned}
$$

The slope $S_{0}$, on the other hand, is in general maximized by a non-uniform power allocation. For sufficiently low SNR, however, $\frac{E_{b}^{r}}{N_{0}}$ min dominates the behavior. The more that the power allocation departs from uniform to improve $S_{0}$, the higher the price paid in terms of $\frac{E_{b}^{r}}{N_{0}}$ min .

\section{E Proof of Proposition 5}

With $n_{\mathrm{T}} \leq n_{\mathrm{R}}$, the slope $S_{\infty}$ is sure to equal $n_{\mathrm{T}}$ if only $p_{j}>0, j=1, \ldots, n_{\mathrm{T}}$. Under that condition, we may focus exclusively on $\mathcal{L}_{\infty}$.

Suppose that the $j$ th codeword is the one limiting the overall spectral efficiency. From (48) and (50),

$$
\begin{aligned}
\mathcal{L}_{\infty}= & \log _{2} n_{\mathrm{T}}-\log _{2} \operatorname{det}\left(\mathbf{P}_{j-1}\right)+\log _{2} \operatorname{det}\left(\mathbf{P}_{j}\right) \\
& -E\left[\log _{2} \operatorname{det}\left(\mathbf{H}_{j-1}^{\dagger} \mathbf{H}_{j-1}\right)\right]+E\left[\log _{2} \operatorname{det}\left(\mathbf{H}_{j}^{\dagger} \mathbf{H}_{j}\right)\right]
\end{aligned}
$$


Exploiting the diagonal forms of $\mathbf{P}_{j-1}$ and $\mathbf{P}_{j}$ and using (46) to evaluate the expectations,

$$
\mathcal{L}_{\infty}=\log _{2} n_{\mathrm{T}}-\log _{2} p_{j}+\left(\gamma-\sum_{\ell=1}^{n_{\mathrm{R}}-n_{\mathrm{T}}+j-1} \frac{1}{\ell}\right) \log _{2} e .
$$

Because of the max-min optimization in (31), the optimum power allocation is the one that results in the same $\mathcal{L}_{\infty}$ for every $j \in\left(1, \ldots, n_{\mathrm{T}}\right)$. Enforcing that condition, as well as the power constraint $\sum_{j=1}^{n_{\mathrm{T}}} p_{j}=n_{\mathrm{T}}$, the optimum power allocation and the corresponding power offset are obtained after a modicum of algebra.

\section{References}

[1] G. J. Foschini, "Layered space-time architecture for wireless communications in a fading environment when using multi-element antennas," Bell Labs Tech. J., pp. 41-59, 1996.

[2] G. D. Golden, G. J. Foschini, R. A. Valenzuela, and P. W. Wolniansky, “Detection algorithm and initial laboratory results using V-BLAST space-time communications architecture," Electronic Letters, Nov. 1998.

[3] G. J. Foschini, D. Chizhik, M. J. Gans, C. Papadias, and R. A. Valenzuela, “Analysis and performance of some basic space-time architectures," IEEE J. Sel. Areas in Communications, vol. 21, pp. 303-320, Apr. 2003.

[4] M. K. Varanasi and T. Guess, "Optimum decision-feedback multiuser equalization with successive decoding achieves the total capacity of the Gaussian multiple-access channel," Asilomar Conference, pp. 1405-1409, 1998.

[5] M. K. Varanasi and T. Guess, "An information-theoretic framework for deriving canonical decision-feedback receivers in Gaussian channels," IEEE Trans. Inform. Theory, vol. 51, no. 1, pp. 173-187, Jan. 2005.

[6] S. T. Chung, A. Lozano, and H. C. Huang, "Approaching eigenmode BLAST channel capacity using V-BLAST with rate and power feedback," IEEE Vehic. Techn. Conf. (VTC'2001 Fall), Atlantic City, NJ, Oct. 2001.

[7] A. Lozano, "Capacity-approaching rate function for layered multi-antenna architectures," IEEE Trans. on Wireless Comm., vol. 2, pp. 616-620, July 2003. 
[8] H. Zhuang, L. Dai, S. Zhou, and Y. Yao, "Low complexity per-antenna rate and power control approach for closed-loop V-BLAST," IEEE Trans. Communications, vol. 51, pp. 1783-1787, Nov. 2003.

[9] N-Prasad and M. K. Varanasi, "Analysis of decision feedback detection for MIMO Rayleighfading channels and the optimization of power and rate allocations," IEEE Trans. Inform. Theory, vol. 50, pp. 1009-1025, June 2004.

[10] S. T. Chung, A. Lozano, H. C. Huang, A. Sutivong, and J. M. Cioffi, "Approaching the MIMO capacity with V-BLAST: theory and practice," EURASIP Journal on Applied Signal Processing (special issue on MIMO), vol. 2002, pp. 762-771, May 2004.

[11] S. Grant, J.-F. Cheng, L. Krasny, K. Molnar, and Y.-P. E. Wang, "Per-antenna-rate-control (PARC) in frequency selective fading with SIC-GRAKE receiver," IEEE Veh. Techn. Conf. (VTC'04), 2004.

[12] A. Milani, V. Tralli, and M. Zorzi, “On the use of rate and power adaptation in V-BLAST systems for data protocol performance improvement," IEEE Trans. Wireless Communications, vol. 5, pp. 16-22, Jan. 2006.

[13] J. Liao, A. Furuskar, P. Skillermark, and B. Goransson, "Impact of transmit antenna separation on downlink cellular OFDM system with PARC transmission," Int'l Symp. on Personal, Indoor and Mobile Radio Communications, 2007.

[14] 3GPP RAN Working Group 1, TR 25.876 v7.0.0, Multiple Input Multiple Output in UTRA (Release 7), 2007.

[15] S. Sesia, I. Toufik, and M. Baker (Editors), The UMTS Long Term Evolution: From Theory to Practice, Wiley, 2009.

[16] J. G. Andrews, A. Ghosh, and R. Muhamed, Fundamentals of WiMAX, Prentice Hall, 2007.

[17] P. Layec, R. Visoz, and A. O. Berthet, "Achieving high spectral efficiency with adaptive layered space time codes under rate control," IEEE Int'l Conf. on Communications (ICC'07), 2007.

[18] J. Li, Z. Zhou, H. Guo, and J. Zhou, “Optimized CQI quantization based PARC scheme for multi-stream MIMO systems," IEEE Wireless Communications and Networking Conference (WCNC'07), 2007.

[19] I. E. Telatar, “Capacity of multi-antenna Gaussian channels," Eur. Trans. Telecom, vol. 10, pp. 585-595, Nov. 1999. 
[20] A. Lozano and N. Jindal, "Transmit diversity v. spatial multiplexing in modern MIMO systems," to appear in IEEE Trans. on Wireless Communications, 2009.

[21] S. Verdú, "Spectral efficiency in the wideband regime," IEEE Trans. on Inform. Theory, vol. 48, no. 6, pp. 1319-1343, June 2002.

[22] S. Shamai and S. Verdú, "The impact of frequency-flat fading on the spectral efficiency of CDMA," IEEE Trans. on Inform. Theory, vol. 47, no. 5, May 2001.

[23] G. J. Foschini, G. D. Golden, R. A. Valenzuela, and P. W. Wolnianski, "Simplified processing for high spectral efficiency wireless communication employing multi-element arrays," IEEE J. Select. Areas Commun., vol. 17, no. 11, pp. 1841-1852, Nov. 1999.

[24] H. Shin and J. H. Lee, "Capacity of multiple-antenna fading channels: Spatial fading correlation, double scattering and keyhole," IEEE Trans. Inform. Theory, vol. 49, pp. 2636-2647, Oct. 2003.

[25] M. Abramowitz and I. A. Stegun, Handbook of Mathematical Functions, New York: Dover Publications, 1972.

[26] A. Lozano, A. M. Tulino, and S. Verdu, "High-SNR power offset in multiantenna communication," IEEE Trans. Inform. Theory, vol. 51, no. 12, pp. 4134-4151, Dec. 2005.

[27] A. M. Tulino, A. Lozano, and S. Verdu, "Impact of correlation on the capacity of multiantenna channels," IEEE Trans. Inform. Theory, vol. 51, pp. 2491-2509, July 2005.

[28] A. Zanella, M. Chiani, and M. Z. Win, "Analytical evaluation of MIMO systems with unequal power transmission in a Rayleigh fading environment," Proc. of IEEE GLOBECOM'03, vol. 4, pp. 1837-1841, Dec. 2003.

[29] A. M. Tulino, A. Lozano, and S. Verdú, "Capacity-achieving input covariance for single-user multi-antenna channels," IEEE Trans. on Wireless Communications, vol. 5, no. 2, pp. 662-671, Mar. 2006.

[30] M. Chiani, M. Z. Win, and A. Zanella, "On the capacity of spatially correlated MIMO Rayleighfading channels," IEEE Trans. Inform. Theory, vol. 49, no. 10, pp. 2363-2371, Oct. 2003.

[31] P. J. Smith, S. Roy, and M. Shafi, "Capacity of MIMO systems with semi-correlated flat fading," IEEE Trans. on Inform. Theory, vol. 49, no. 10, pp. 2781-2788, Oct. 2003. 
[32] M. Kiessling, "Unifying analysis of ergodic MIMO capacity in correlated Rayleigh fading environments," Eur. Trans. Telecom., vol. 16, no. 1, pp. 17-35, Jan. 2005.

[33] H. Shin, M. Z. Win, J. H. Lee, and M. Chiani, “On the capacity of doubly correlated MIMO channels," IEEE Trans. Wireless Communications, vol. 5, no. 8, pp. 2253-2265, Aug. 2006.

[34] R. J. Muirhead, Aspects of multivariate statistical theory, New York, Wiley, 1982. 
Figure 1: MIMO layered architecture transmitter with $n_{\mathrm{T}}$ encoders, each feeding an antenna. 
Figure 2: Low-power spectral efficiencies of PARC and H-BLAST with $n_{\mathrm{T}}=n_{\mathrm{R}}=4$. In solid, exact values. In dashed, low-power expansions with the performance measures in (18)-(19) and in Proposition 1, respectively. 
Figure 3: Low-power spectral efficiencies of PARC and H-BLAST with $n_{\mathrm{T}}=4, n_{\mathrm{R}}=6$. In solid, exact values. In dashed, low-power expansions with the performance measures in (18)-(19) and in Proposition 1, respectively. 
Figure 4: High-power spectral efficiencies of PARC and H-BLAST with $n_{\mathrm{T}}=n_{\mathrm{R}}=4$. In solid, exact values. In dashed, high-power expansions with $S_{\infty}=4$ and with the power offsets in (24) and in Proposition 3, respectively. 
Figure 5: High-power spectral efficiencies of PARC and H-BLAST with $n_{\mathrm{T}}=4, n_{\mathrm{R}}=6$. In solid, exact values. In dashed, high-power expansions with $S_{\infty}=4$ and with the power offsets in (24) and in Proposition 3, respectively. 
Figure 6: Exact high-power spectral efficiencies of PARC and H-BLAST with $n_{\mathrm{T}}=n_{\mathrm{R}}=4$ and uniform power allocations. Also shown is the corresponding spectral efficiency for H-BLAST, likewise exact, with the power allocation given in Proposition 5. 